\title{
Personalization, Socialization, Contextualization: Preferences and Attitudes for Advanced Information Provision
}

\author{
Yannis Ioannidis \\ Dept. of Informatics and Telecommunications \\ National and Kapodistrian University of Athens
}

Human actions in real life are often influenced by several characteristics of the individual human involved in the actions. These characteristics can be broadly classified into three categories: those that are unique to the individual, those of the social environment of the individual, and those of the overall context or situation in which the individual is found while performing the actions. Usability of various types of information systems, e.g., database systems, digital libraries, or the Web, increases dramatically if the information they provide and their overall behavior is customized to these characteristics. Such personalization, socialization, and contextualization of information provision touches upon a broad spectrum of technical and other challenges.

This talk describes the general problem and its associated challenges, hints upon a general framework for modeling a large number of cases, and offers some examples of systems and techniques that have been developed by the Univ. of Athens to address related challenges in various application environments. 